\title{
The Air Defense of Ship Formation Decision-making Optimization
}

\author{
Ma Liang \\ China Department of Surface Ship Command \\ Dalian Naval Academy \\ Dalian, China \\ E-mail: $446982051 @ q q . c o m$
}

\begin{abstract}
- the air defense of ship formation cooperation decision-making is a multi-stage multi-objective system. Based on the concepts of the fuzzy weighted distance and membership degree, considering the collaborative relationship in different phase, put forward the concept of thermodynamic power allotment wreath of the cooperation degree,combined the fuzzy optimum selection theory with dynamic programming methodology,it provides a new method for solving the multi-stage multi-objective optimization based on collaboration to obtain the optimum balanced tactic. This method is applied to solve the collaborative decision-making optimization problem of colligation anti-missile by the air defense of ship formation cooperation and gives some instructive results.
\end{abstract}

Keywords-thermodynamic power allotment wreath; cooperation degree; optimization; dynamic programming methodology

\section{INTRODUCTION}

Ships formation cooperative anti-air is a multi-stage and multi-objective decision-making system based on collaborative. The anti-air combat effect and combat resource consumption is a pair of contradiction. Operational effectiveness use minimal resource consumption to gain the biggest operational effects. For different decision form different operational effectiveness, operational effectiveness on surface is different, have different resource allocation, it is a complicated problem. Comprehensive analysis of fleet anti-missile process, we can see that it is a multi-stage multi-objective system, and because there is synergy between the stages, so we need to search based on multi-stage multi-objective dynamic optimization method of synergy. Multi-stage system optimization objective is to seek to optimize the index of the whole process of the target or the decision strategy of each stage. Fuzzy distance and membership degree is presented in this paper on the basis of the concept of coordination degree on the basis of fire distribution ring measurement, the fuzzy optimization theory and dynamic programming theory organically, and based on

\author{
Zhang Lin \\ Department of Missile \\ Dalian Naval Academy \\ Dalian, China \\ E-mail: mailbox1015@126.com
}

coordination degree of fire distribution ring multi-stage multi-objective decision-making system fuzzy optimization dynamic programming model.

\section{II .THE CONCEPT OF COORDINATION DEGREE OF FIRE DISTRIBUTION RING}

\section{A The concept of fire distribution ring}

In the surface warship's anti-air combat, the ship formation can carry on the fire distribution to ensure the effective interception target, when the target in the kill zone range ship to air missile weapon system. If early fire distribution in far field emission region, on the one hand may be due to the target maneuver to abandon attempted attack, and the fire distribution is effective; On the other hand, easy to cause fire in the process of air defense combat premature transfer, thereby reducing firing efficiency. If later than the launch area close to fire distribution, ship to air missile weapon system will not be able to normal play, causing the target under the condition of the force against penetration. Will be launched from a certain height on the corresponding area near boundary, the far field emission region of area in a horizontal projection called fire distribution on the surface of the ring ${ }^{[1]}$.

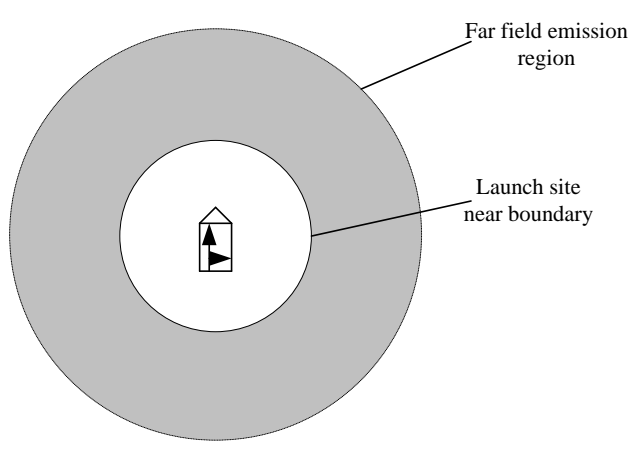

Figure 1. fire distribution ring concept sketch

\section{B Fire distribution window of opportunity}

Fire distribution opportunity window refers to the target into the formation of empty fire distribution ring moment, to the left wing fire distribution ring moment of time. In the process of formation of air defense combat, 
goals must be efficient allocated during this period, that is to say, fleet commander must be within the window of opportunity to make allocation decisions, or goals will leave the ring of fire distribution, penetration and to achieve.

$t^{i}$ In one firepower unit, the unit window of opportunity $t_{m}$ is the $i$ firepower to $m$ for $_{i}$ the target, the target must be in this period of time $t_{m}$ is allocated to the firepower unit, otherwise the firepower unit cannot be assigned to the target, the target will break through the layer of defense.

For formation fire distribution ring, the two firepower unit between two ship fire distribution opportunity window on the lateral, or both horizontal and vertical overlap in formation when fire distribution must be coordinated. The coordinate methods, available firepower unit coordination degree.

$j$ When the target $m$ into the firepower unit $i$ and $J$ collaboration on the ring domain, might be both a firepower unit service, if each service success probability high enough firepower unit is a waste, sometimes will lose the best chance to intercept more goals; If each firepower unit service success probability is too low, it will improve the probability of intercept, but if the two firepower unit for lack of information communication between decisions alone, can cause depend on each other, make if coordinated well can be intercepted target, without intercept. For the collaboration domain goal must have a consensus on the unified allocation decisions.

\section{Fire distribution ring synergy degree}

The target window of opportunity $t_{m}^{i}$ is the firepower $i$ unit to the target $m$, the target window of opportunity $t_{m}^{j}$ is the firepower unit $j$ to the target $m$, the target in collaboration domain is $\Delta t_{m}$.

Make for firepower unit and collaborative coefficient, the coordination degree of the matrix firepower unit $i$ and $j$ is:

$X T_{i j}=\left[\begin{array}{ll}0 & \alpha_{i j} \\ \alpha_{j i} & 0\end{array}\right] \alpha_{i j} \in[0,1] \alpha_{i j}=\Delta t_{m} / t_{m}^{i} \alpha_{j i}=\Delta t_{m} / t_{m}^{j}$

(1) If $\alpha_{i j}=0(i \neq j)$, the firepower unit $i$ and firepower unit $j$ have no collaboration, their respective fire distribution ring is isolated; If any $\alpha_{i j}=0(i \neq j)$, the ring formation doesn't exist in coordination, collaboration domain is empty, the formation of all the air defense firepower unit will be shooting independently, without collaboration.

(2) If $\alpha_{i j}=1(i \neq j)$, the firepower unit $i$ and firepower unit $j$ have collaboration relationship, and any firepower units are assigned to the target with the same time effect.

(3) If $0<\alpha_{i j}<1(i \neq j)$ the firepower unit $i$ and firepower unit $j$ have collaboration relationship, and any firepower units are assigned to the target with different time effect. If $\alpha_{i j}>\alpha_{j i}$ the firepower unit $i$ shot on the target time is less than firepower unit $j$ shooting time of the target.

\section{MULTIPLE TARGET DECISION OPTIMIZATION PHASE} SYSTEM

A multi-stage system contains $K$ phases and $m$ targets, using the forward dynamic programming method. From phase one, backward recursive sequence, for multi-objective optimization problem, the recursive formula can be represented as:

$$
F_{k}\left(S_{k}^{t}\right)=\operatorname{opt}\left\{F_{k-1}\left(S_{k-1}^{t}\right) \oplus H_{k}\left(S_{k}, d_{k}\right)\right\}
$$

The equation of state for:

$$
S_{k}=\varphi_{k}\left(S_{k-1}, d_{k}\right) \quad k=1,2, \cdots, K
$$

$F_{k}\left(S_{k}^{t}\right)=\left[f_{k}^{(1)}\left(S_{k}^{t}\right), \cdots, f_{k}^{(m)}\left(S_{k}^{t}\right)\right]^{T}$,

$F_{k-1}\left(S_{k-1}^{t}\right)=\left[f_{k-1}^{(1)}\left(S_{k-1}^{t}\right), \cdots, f_{k-1}^{(m)}\left(S_{k-1}^{t}\right)\right]^{T}$, Respectively the

local optimal state $S_{k}^{t}, S_{k-1}^{t}$ tentatively determined in phase $k$ and $k-1$ as a target of synthetic value vector; $H_{k}\left(S_{k}, d_{k}\right)=\left[h_{k}^{(1)}\left(S_{k}, d_{k}\right), \cdots, h_{k}^{(m)}\left(S_{k}, d_{k}\right)\right]^{T}$ is phase $k$ state values $S_{k}$ for decision-making $d_{k}$ when $m$ project consisting of a vector. Superscript $t$ on behalf of the provisional optimal, $\oplus$ as the target composition operator.

Forward recursive, the corresponding $n_{k}$ strategy, $m$ goals, the phase $k$ state $S_{k}$ is a synthetic value matrix when the target is:

$V_{k}\left(S_{k}\right)=\left|\begin{array}{ccc}v_{11}\left(S_{k}\right) & \cdots & v_{1 n}\left(S_{k}\right) \\ v_{21}\left(S_{k}\right) & \cdots & v_{2 n}\left(S_{k}\right) \\ \vdots & & \vdots \\ v_{m 1}\left(S_{k}\right) & \cdots & v_{m n}\left(S_{k}\right)\end{array}\right| v_{i j}\left(S_{k}\right)=f_{k-1}^{(i)}\left(S_{k-1}^{t}\right) \oplus h_{k}^{(i)}\left(S_{k}, d_{j}\right)$

Synthesize target value matrix $V_{k}\left(S_{k}\right)$ optimal normalized to the corresponding matrix, the formula can be used ${ }^{[2]}$ :

$$
r_{i j}\left(S_{k}\right)=\mid \begin{gathered}
\frac{v_{i j}\left(S_{k}\right)}{\max _{j} v_{i j}\left(S_{k}\right)} \\
1-\frac{v_{i j}\left(S_{k}\right)}{\max _{j} v_{i j}\left(S_{k}\right)}
\end{gathered} \text { the bigger the optimal type }
$$

Synthesize target value standardization respectively. $r_{i j}\left(S_{k}\right)$ expressed the strategy $v_{i j}\left(S_{k}\right)$ of the $i$ item target synthetic value for optimal membership degree, $\max v_{i j}\left(S_{k}\right)$ referred to as target membership degree to take a strategy $n_{k}$ of maximum synthesis the $i$ target. The membership degree matrix: 


$$
R_{k}\left(S_{k}\right)=\left|\begin{array}{ccc}
r_{11}\left(S_{k}\right) & \cdots & r_{1 n_{k}}\left(S_{k}\right) \\
r_{21}\left(S_{k}\right) & \cdots & r_{2 n_{k}}\left(S_{k}\right) \\
\vdots & & \vdots \\
r_{m 1}\left(S_{k}\right) & & r_{m n_{k}}\left(S_{k}\right)
\end{array}\right|
$$

Make relatively superior strategy $g\left(S_{k}\right)$ for:

$$
g\left(S_{k}\right)=\left(g_{1}\left(S_{k}\right), \cdots, g_{m}\left(S_{k}\right)\right)=\left(\underset{j}{\vee} r_{i j}\left(S_{k}\right), \cdots, \underset{j}{v} r_{m j}\left(S_{k}\right)\right)
$$

Relatively inferior strategy $b\left(S_{k}\right)$ for:

$$
\begin{aligned}
& b\left(S_{k}\right)=\left(b_{1}\left(S_{k}\right), \cdots, b_{m}\left(S_{k}\right)\right)=\left(\wedge_{j} r_{i j}\left(S_{k}\right), \cdots, \wedge r_{j} r_{m j}\left(S_{k}\right)\right) \\
& \vee \underset{j}{\vee} r_{i j}\left(S_{k}\right)=r_{i 1}\left(S_{k}\right) \vee r_{i 2}\left(S_{k}\right) \vee \cdots \vee r_{i n_{k}}\left(S_{k}\right) \\
& \hat{j}_{j} r_{i j}\left(S_{k}\right)=r_{i 1}\left(S_{k}\right) \wedge r_{i 2}\left(S_{k}\right) \wedge \cdots \wedge r_{i n_{k}}\left(S_{k}\right)
\end{aligned}
$$

The importance of the optimization goal $m$ set item weight vector is:

$$
w=\left(w_{1}, w_{2}, \cdots, w_{m}\right), \quad \sum_{i=1}^{m} w_{i}=1
$$

The strategy $v_{j}\left(S_{k}\right)$ goal membership vector is: $r_{j}\left(S_{k}\right)=\left(r_{1 j}\left(S_{k}\right), \cdots, r_{m j}\left(S_{k}\right)\right)$

The strategies $v_{j}\left(S_{k}\right)$ and the relative advantage $g\left(S_{k}\right)$ difference between master and relatively inferior strategy $b\left(S_{k}\right)$, respectively, by right of generalized distance can be expressed as ${ }^{[6]}$ :

$$
\begin{aligned}
\| w\left[g\left(S_{k}\right)-r_{j}\left(S_{k}\right)\right] \mid & =\sum_{i=1}^{m}\left\{w_{i}\left[g_{i}\left(S_{k}\right)-r_{i j}\left(S_{k}\right)\right]\right\} \\
\left\|w\left[r_{j}\left(S_{k}\right)-b\left(S_{k}\right)\right]\right\| & =\sum_{i=1}^{m}\left\{w_{i}\left[r_{i j}\left(S_{k}\right)-b_{i}\left(S_{k}\right)\right]\right\}
\end{aligned}
$$

Set the strategy $v_{j}\left(S_{k}\right)$ Optimal in "Optimal" belongs to the fuzzy subset $u_{j}\left(S_{k}\right)$ to inferior degree "Carrier", belongs to the fuzzy subset $u_{j}^{c}\left(S_{k}\right)$ based on fuzzy set are defined

$u_{j}^{c}\left(S_{k}\right)=1-u_{j}\left(S_{k}\right)$

According to the optimal principle, the corresponding strategies $\max \left\{u_{j}\left(S_{k}\right)\right\}$ available interim optimal decision $d_{k}^{t}$ is the state $S_{k}$ of phase $k$, and at the same time the tentative status $S_{k}^{t}$ is the optimal state, the optimal tentative target resultant vector is obtained at the same time ${ }^{[4]}$.

$$
F_{k}\left(S_{k}^{t}\right)=\left[f_{k}^{(1)}\left(S_{k}^{t}\right), \cdots, f_{k}^{(m)}\left(S_{k}^{t}\right)\right]^{T}
$$

Because phase 1 has $L$ tentative optimal states, so in the final $K$ stage of target synthetic value matrix of the:

$$
\begin{gathered}
F_{K}\left(S_{K}\right)=\left|\begin{array}{ccc}
f_{k}^{(1)}\left(S_{k}^{1}\right) & \cdots & f_{k}^{(1)}\left(S_{k}^{L}\right) \\
f_{k}^{(2)}\left(S_{k}^{1}\right) & \cdots & f_{k}^{(2)}\left(S_{k}^{L}\right) \\
\vdots & \vdots \\
f_{k}^{(m)}\left(S_{k}^{1}\right) & & f_{k}^{(m)}\left(S_{k}^{L}\right)
\end{array}\right| \\
f_{k}^{(i)}\left(S_{k}^{t}\right)=v_{i t}\left(S_{k}\right) \text {, using the above method and }
\end{gathered}
$$

the model type (13) and the stage of $K$ is the optimal state of the provisional membership degree, the corresponding state $\max \left\{u_{t}\left(S_{k}^{*}\right)\right\}$ is real global optimal state $S_{k}^{*}$. At the same time to obtain corresponding real global optimal target value vector ${ }^{[5]}$ :

$$
F_{K}\left(S_{K}^{*}\right)=\left[f_{k}^{(1)}\left(S_{K}^{*}\right), \cdots, f_{k}^{(m)}\left(S_{K}^{*}\right)\right]^{T}
$$

And in each stage of the optimal strategy of the whole system is the optimal decision of collection $d_{1}^{*}, d_{2}^{*}, \cdots, d_{K}^{*}$.

When the target weight vector $w$ to determine, using the multi-objective multistage process based on collaborative optimization fuzzy optimization dynamic programming technology can get an optimal solution is a non inferior solution of multi-objective optimization problem. If changed in accordance with the goal of the relative importance of different target weight vector $w$, the system can be obtained accordingly non inferior solution of multi-objective optimization, for policymakers to choose according to situation is satisfactory solutions.in the optimal equilibrium strategy of system optimization.

\section{THE EXAMPLE ANALYSIS}

In the ship formation of cooperative anti-missile, it can be divided into three phases: medium-range ship to air missile, medium and near-range ship to air missile and near-range ship to air missile. It can be seen that the tf synergy anti-missile system is not only more stage, is also a multiple objective system. In the process of antimissile, hope not only formation cooperative anti-missile interception probability is high, but also want to use the resources of as little as possible, and to facilitate sustained combat, so the system is a three stage binocular mark dynamic decision-making system.

According to statistics, in all phases of the fleet anti-missile together in a missile intercept probability and its operational resources consumed in table 1 (in parentheses for decision-making status number), set the ship to air missile launching area system is shown in table 2. 


\begin{tabular}{|c|c|c|c|c|c|}
\hline \multirow{4}{*}{ medium-range ship to air missile } & \multirow{2}{*}{ index function } & \multicolumn{4}{|c|}{ distribution of firepower unit number } \\
\hline & & $1(1)$ & $2(2)$ & $3(3)$ & $4(4)$ \\
\hline & intercept probability & 0.42 & 0.66 & 0.80 & 0.89 \\
\hline & Resource consumption & 0.2 & 0.4 & 0.6 & 0.8 \\
\hline \multirow{4}{*}{$\begin{array}{c}\text { medium and near-range ship to } \\
\text { air missile }\end{array}$} & & \multicolumn{4}{|c|}{ distribution of firepower unit number } \\
\hline & index lunction & \multicolumn{2}{|c|}{$1(5)$} & \multicolumn{2}{|c|}{$2(6)$} \\
\hline & intercept probability & \multicolumn{2}{|c|}{0.67} & \multicolumn{2}{|c|}{0.89} \\
\hline & Resource consumption & \multicolumn{2}{|c|}{0.25} & \multicolumn{2}{|c|}{0.5} \\
\hline \multirow{3}{*}{ near-range ship to air missile } & index function & \multicolumn{4}{|c|}{$1(7)$} \\
\hline & intercept probability & \multicolumn{4}{|c|}{0.75} \\
\hline & Resource consumption & \multicolumn{4}{|c|}{0.25} \\
\hline
\end{tabular}

TABLE II. SHIP TO AIR MISSILE LAUNCHING AREA AND COMMUNITY SETTINGS

\begin{tabular}{ccc}
\hline Serial number & Launch site near boundary $(\mathrm{km})$ & Far field emission region $(\mathrm{km})$ \\
\hline ship to air missile 1 & 9 & 35 \\
\hline ship to air missile 2 & 6 & 20 \\
\hline ship to air missile 3 & 3 & 9 \\
\hline
\end{tabular}

According to the formula (1) and table 2 data calculating synergy degree matrix is as follows:

$$
X T=\left[\begin{array}{lll}
0 & 0.42 & 0 \\
0.79 & 0 & 0.21 \\
0 & 0.38 & 0
\end{array}\right]
$$

This is a 3 phase collaborative decision-making system, only consider the former stage on the last stage of coordinated degree, in order that is now to be solved by recursion. In each stage of operation at the end of the penetration probability of anti-ship missile and fleet combat resource depletion as each phase of the output. In the beginning, the penetration probability of missile should be 1 , combat resource depletion is 0 , because at this time had not yet taken countermeasures of missile, and therefore no fighting began to consume resources. The decision variables is $d_{k}$ for the operation. System operational resources of total consumption of the optimization goal 1 , take up the synthesis operator $\oplus$; Missile penetration probability as the optimization goal 2 , according to the nature of the target composition operator $^{[6]}$ :

$$
\oplus=\left(1-H_{k}\left(S_{k}, d_{k}\right)\right) \times F_{k-1}\left(S_{k-1}^{t}\right) \quad, \quad \text { Synthetic }
$$

operator $\otimes$ take multiply in this case, the lower the next phase of the missile interception probability. According to the nature of the two objectives are the smaller type. The goal is as important as the two, namely target type $w=(0.5,0.5)^{T}$.
(1) In the stage $K=1$, medium range ship to air missile combat. After this phase, the missile penetration probability and combat resource depletion as follows:

$$
\begin{gathered}
F_{1}(1)=(1,0) \oplus(0.42,0.2)=(0.58,0.2) \\
F_{1}(2)=(1,0) \oplus(0.66,0.4)=(0.34,0.4) \\
F_{1}(3)=(1,0) \oplus(0.8,0.6)=(0.2,0.6) \\
F_{1}(4)=(1,0) \oplus(0.89,0.8)=(0.11,0.8)
\end{gathered}
$$

(2) In the stage $\mathrm{K}=2, F_{1}(1)$ is now in phase 1 tentatively for optimal decision, the intermediate and short-range ship to air missile intercepting phase, are:

$$
F_{2}\left(S_{2}^{1}\right)=\left|\begin{array}{cc}
0.58 & 0.58 \\
0.2 & 0.2
\end{array}\right| \oplus\left|\begin{array}{cc}
0.67 & 0.89 \\
0.25 & 0.5
\end{array}\right| \otimes 0.79=\left|\begin{array}{cc}
0.0401 & 0.0133 \\
0.608 & 0.858
\end{array}\right|
$$

(3) In the stage $\mathrm{K}=3$, the solving process as (2). For close range of ship to air missile interceptor phase, are:

$$
F_{3}\left(S_{3}^{1}\right)=\left|\begin{array}{cc}
0.0401 & 0.0133 \\
0.608 & 0.858
\end{array}\right| \oplus\left|\begin{array}{c}
0.75 \\
0.25
\end{array}\right| \otimes 0.38=\left|\begin{array}{cc}
0.0062 & 0.002 \\
1.089 & 1.434
\end{array}\right|
$$

Tentative respectively, $F_{1}(2) F_{1}(3) F_{1}(4)$ for the most decisions, solving process is alexandrine available:

$$
\begin{aligned}
& F_{3}\left(S_{3}^{2}\right)=\left|\begin{array}{rr}
0.0036 & 0.0012 \\
1.583 & 1.928
\end{array}\right| 、 F_{3}\left(S_{3}^{3}\right)=\left|\begin{array}{ll}
0.0021 & 0.0007 \\
2.0771 & 2.4221
\end{array}\right| \text { 、 } \\
& F_{3}\left(S_{3}^{4}\right)=\left|\begin{array}{ll}
0.0011 & 0.0003 \\
2.5711 & 2.9161
\end{array}\right|
\end{aligned}
$$

On the basis of matrix $F_{3}\left(S_{3}\right)$ final optimization global optimal real status is $S_{3}^{*}$, the application of the 
above method and the fuzzy optimization model (13) in the final stages of type 4 tentative optimal state and synthetic value of membership goals: $u\left(S_{3}^{t}\right)=(0.21,0.71,0.73,0.69)$

Optimal with the maximum 0.73 corresponding state $S_{3}^{3}=3-5-7$ is the global optimal real state, and the corresponding real global optimal synthesis value vector is: $F_{3}\left(S_{3}^{*}\right)=(0.00105,1.03855)^{T}$, namely when the fleet anti-missile together, balance attaches great importance to the effect of abm and combat resource consumption, best way to response of the system. At this point, the missile penetration probability is only $0.105 \%$, and combat resource consumption is only 1.038 .

\section{CONCLUSION}

Based on multi-stage optimization decision-making coordination of multi-objective problem of tf synergy anti-missile decision makes sense. In the current vessel anti-missile limited resources, intercept probability of a certain situation, how to improve the operational effectiveness of fleet anti-missile together, improve the comprehensive capability of formation, is the research hotspot and difficult problem of air defense missile. Based on the fuzzy dynamic programming on the basis of solving the multi-stage multi-objective optimization decision-making, introduced based on the concept of fire distribution ring coordination degree, the collaborative relationship between the formation weapon system is described, and the solution is effectively used in the optimization process, based on coordinate to solve multi-stage multi-objective optimization decision-making problem provides a new way. But this paper is just a preliminary study, the discusses the tf firepower together against the simplest case, target for incoming flow and fleet fire collaborative network influence on missile defense, this article does not take into account, the follow-up work will be further research.

\section{REFERENCES}

[1]Galay Barbarosoglu, Linet Ozdamar, Ahmel Cevik.An Interactive Appmach of Hierarchical Analysis of Helicopter Logistics in Disaster Relief Operations[J]. European Journal of Operational Research, $2002: 118-133$

[2]WANG Hong-jun, CHI Zhong-xian.Shipboard hard and soft weapon anti-missile decision-makingoptimization based on collaboration[J], Control and Decision, 2007, 22 (3) : 299-303

[3]Liang Bo, Duan Ran.Fire distribution model based on feasible direction algorithm $[\mathrm{J}]$, Command Information System and Technology, 2013,4 (2) :30-32

[4]L Ozdamar . Emergency logistics planning in natural disasters[J]. Annal of Operation Research, 2004, 129(11) : 218-219.

[5]Sarit Kraus.Negotiation and cooperation in mulfi-ageat environments.Artificial Intelligence. 1997 : 79-97

[6]HUANG Haixin, WANG Ding wei.English auction dynamic model based on fuzzy game[J], Information and Control,2012,41(4):509-513

[7]Shao Qiu feng, Ma ya long, hu jin chuan.Applied research for fuzzy composite operators in operational alternatives integrated evaluation[J],Journal of Academy of Armored Force Engineering, 2004, 18 (12) : 41-43

[8]LI Zi fen, LI Xiang min, CHEN Jin zhu.Dynamic joint fire distribution method based on decentralized cooperative auction algorithm[J],Fire Control \& Command Control , 2012,37 (11) :50-52

[9]NIAN Song lei, YAN Jian gang, CHEN Rong.The optimization method of antiship missile fire distribution based on dynamic programming[J],Ship Science And Technology, 2012,34 (7) :110-113

[10]MA Liang , QING Zuo-sheng , ZHANG Lin.Formation comprehensive cooperative air defense multi-stage decision optimization modeling[J], Tactical Missile Technology, 2013,9 (5) :25-28

[11]LIU Wei dong, JIANG Qing shan, LI Yong.Fire distribution of the network centric ship-to-air missile based on earlier damage[J],Ship Science And Technology, 2011,33 (2) :98-101

[12]CHEN Guo sheng, JIA Zi ying.Research on coordinated air defense firepower distribution model for warships[J], Command ControI\& Simulation, 2011,33 (12) :13-15 